\title{
Interplay between a crystal's shape and spatiotemporal dynamics in a spin transition material
}

\author{
Houcem Fourati ${ }^{\text {ab }}$, Eric Milin ${ }^{c}$, Ahmed Slimani ${ }^{d}$, Guillaume Chastanet ${ }^{\mathrm{e}}$, Younes Abid ${ }^{\mathrm{b}}$, Smail Triki ${ }^{\mathrm{c}}$ and \\ Kamel Boukheddaden *a
}

${ }^{a}$ Groupe d'Etudes de la Matière Condensée, Université de Versailles, Université Paris-Saclay, CNRS UMR CNRS 8635,45 Avenue des Etats Unis, 78035 Versailles, France.E-mail: kamel.boukheddaden@uvsq.fr

${ }^{b}$ Laboratoire de Physique Appliquée, Faculté des Sciences de Sfax, Université de Sfax, Route de Soukra km 3.5, 3000, Sfax, Tunisia

'Laboratoire de Chimie, Electrochimie Moléculaires, Chimie Analytique, UMR CNRS 6521-Université de Brest, 6 Av. V. Le Gorgeu, CS 93837, 29238 Brest, France

'Laboratoire des Matériaux Multifonctionnels et Applications, Département de physique, Faculté des Sciences de Sfax, Université de Sfax, Route de la Soukra km 3.5, B.P. $n^{\circ} 1171$ - 3000, Sfax, Tunisia

eInstitut de Chimie de la Matière Condensée de Bordeaux, Université de Bordeaux, 87 Av. Doc. A. Schweitzer, F-33608 Pessac, France

+ Electronic supplementary information (ESI) available. See DOI: 10.1039/c8cp00868j

\begin{abstract}
We investigated by means of optical microscopy (OM) the spatiotemporal features of the thermo-induced spin transition of $\left[\mathrm{Fe}(2 \text {-pytrz })_{2}\left\{\mathrm{Pd}(\mathrm{CN})_{4}\right\}\right] \cdot 3 \mathrm{H}_{2} \mathrm{O}$ (1) (2-pytrz $=4$-(2-pyridyl)-1,2,4,4 $\mathrm{H}$-triazole) single crystals having two different shapes (triangle and rectangle). While magnetic and calorimetric measurements, performed on a polycrystalline material, showed the respective average heating and cooling transition temperatures of $\left(T^{\text {down }}{ }_{1 / 2} \sim\right.$ $\left.152 \mathrm{~K}, \mathrm{~T}^{\mathrm{up}}{ }_{1 / 2} \sim 154 \mathrm{~K}\right)$ and ( $\mathrm{T}^{\text {down }}{ }_{1 / 2} \sim 160.0 \mathrm{~K}, \mathrm{~T}^{\text {up }}{ }_{1 / 2} \sim 163.5 \mathrm{~K}$ ), OM studies performed on a unique single crystal revealed significantly different switching temperatures ( $T^{\text {down }_{1 / 2}} \sim 152 \mathrm{~K}$ and $T^{\mathrm{up}} 1 / 2 \sim 162 \mathrm{~K}$ ). OM investigations showed an interface spreading over all crystals during the spin transition. Thanks to the color contrast between the low-spin (LS) and the high-spin (HS) states, we have been able to follow the real time dynamics of the spin transition between these two spin states, as well as access the thermal hysteresis loop of each single crystal. After image processing, the HS-LS interface's velocity was carefully estimated in the ranges [4.4-8.5] $\mu \mathrm{m} \mathrm{s}^{-1}$ and [2.5-5.5] $\mu \mathrm{m}$ $\mathrm{s}^{-1}$ on cooling and heating, respectively. In addition, we found that the velocity of the interface is shape-dependent, and accelerates nearby the crystal's borders. Interestingly, we observed that during the propagation process, the interface optimizes its shape so as to minimize the excess of elastic energy arising from the lattice parameter misfit between the LS and HS phases. All of these original experimental results are well reproduced using a spatiotemporal model based on the description of the spin-crossover problem as a reaction diffusion phenomenon.
\end{abstract}

\section{Introduction}

Spin-crossover ( $\mathrm{SCO}$ ) compounds are typical examples of compounds exhibiting a first-order phase transition with a hysteresis loop. ${ }^{1-3} \mathrm{Fe}(\mathrm{II})$ based SCO complexes are among the most studied switchable molecular materials in which the spin transition takes place between two spin states, namely the high spin (HS) state, stable at high temperature, and the low-spin (LS) state, which is stable at lower temperature. In the former state, the electrons of the $d^{6}$ electronic configuration occupy all orbitals $\left(\mathrm{t}^{4}{ }_{2 \mathrm{~g}} \mathrm{e}_{\mathrm{g}} \mathrm{g}\right)$ according to Hund's rule and the spin moment reaches its maximum value $(S=2)$, while in the latter state the six electrons occupy the fundamental state $\left(\mathrm{t}^{6}{ }_{2 \mathrm{~g}} \mathrm{e}_{\mathrm{g}}^{0}\right)$, thus leading to a null spin moment $(S=0)$. Since the $\mathrm{e}_{\mathrm{g}}$ orbital is known to be an antibonding orbital, it follows that in the HS state the bonding between the Fe and the surrounding nitrogen atoms is weakened, which leads to the increase of the Fe-ligand distance by $10 \%$ compared to that of the LS state, causing a local volume expansion. ${ }^{4}$ 
Consequently, the HS state is softer than the LS one. It is important to mention for the non-specialist reader that the SCO solids are paramagnetic in the HS state and diamagnetic in the LS state. The reason of the absence of magnetic ordering in these systems is mainly due to their molecular structure which rarely leads to covalent bonding between the SCO units and subsequently leads to a large distance $(\sim 1 \mathrm{~nm})$ between the iron sites. From the experimental point of view, the spin transition can be triggered by various external stimuli, such as pressure, light, temperature, and a magnetic field. ${ }^{1,5,6}$ The spin transition is intrinsically a vibronic problem ${ }^{7-10}$ in which the electronic and vibrational structures of the molecule are strongly intricate. The change of the magnetic state of the metal center is accompanied by local volume changes at the molecular level, which delocalizes far from the epicenter of the transformation (long-range interaction), a result of the elastic interactions between the SCO units, leading to a macroscopic volume change, stabilizing the LS and HS phases at low- and high-temperature, respectively. These large volume and electronic changes affect significantly the physical properties of the SCO materials, such as color, mechanical properties, etc. Due to these important changes, several experimental techniques have been used to monitor and study this phenomenon, such as differential scanning calorimetry, ${ }^{11}$ magnetometry, ${ }^{12} \mathrm{X}$-ray diffraction, ${ }^{13}$ Mössbauer spectroscopy, ${ }^{14}$ diffuse reflectivity ${ }^{15}$ and quite recently, optical microscopy (OM). ${ }^{16-22}$ Indeed, $\mathrm{OM}$ is one of the techniques allowing non-destructive observation, and direct imaging of the thermally-induced spin transition, with good spatial $(\sim 0.3 \mu \mathrm{m})$ and temporal (up to $0.01 \mathrm{~s}$ ) resolutions. This technique has the advantage of revealing the spatiotemporal aspects of the spin transition on a selected single crystal, which constitutes major progress in the study and understanding of the fundamental mechanisms at the origin of the dynamics of the spin transition, particularly in the case of slow kinetics of the phase transition, which is often the case when the latter occurs with an important volume change.

A variety of SCO compounds have been studied by OM. ${ }^{16-22}$ In ref. 20 for example, the first experiments that we have conducted concerned the brittle single crystals of $\left[\mathrm{Fe}(\mathrm{btr})_{2}(\mathrm{NCS})_{2}\right] \mathrm{H}_{2} \mathrm{O}$ (btr $=4,4^{\prime}$-bis-1,2,4-triazole), whose studies have been performed by placing the single crystals in oil to avoid dehydration. Despite such protection, the first cooling process from HS to LS induces several cracks in the crystal because of mechanical stresses generated by the volume change at the spin transition, which prevented a detailed study of their spatiotemporal properties. However, it was possible to estimate the front velocity on cooling, which was found to be around $2.3 \mu \mathrm{m} \mathrm{s}^{-1}$. In ref. 21, C. Chong et al. studied [ $\left.\mathrm{Fe}(\mathrm{bbtr})_{3}\right]\left(\mathrm{ClO}_{4}\right)_{2}$ single crystals characterized by an hexagonal shape. Likewise, the spin transition of the latter crystals revealed a neat interface that adapts its length and shape along its motion, but unfortunately the crystals always break down after the first cooling, and it was impossible to study the heating branch. The reason of this breaking is due to the large misfit of lattice parameters between the LS and HS states which induces large strains around the interface regions as also discussed in the theoretical electro-elastic models devoted to this phenomenon. ${ }^{22-26}$ More recent studies, performed on [\{Fe(NCSe)(py $\left.)_{2}\right\}_{2}(\mu$-bpypz)] $(p y=$ pyridine and bpypz $=3,5$-bis(2-pyridyl)-pyrazolate) single crystals ${ }^{22,26,27}$ showed a robust system in which it was possible to follow by OM the propagation of the HS/LS front interface on cooling and heating. The velocities were estimated in the interval 4-6 $\mathrm{m} \mathrm{s}^{-1}$. Due to the regular form of the crystal, as elongated platelets along the direction of the interface propagation, the shape of the HS/LS interface remains invariant along the spin transition process.

In the current study, we present new and original optical investigations, conducted on a robust and resilient spincrossover single crystal of formula $\left[\mathrm{Fe}(2 \text {-pytrz })_{2}\left\{\mathrm{Pd}(\mathrm{CN})_{4}\right\}\right] \cdot 3 \mathrm{H}_{2} \mathrm{O}$ (1) $(2$-pytrz $=4$-(2-pyridyl)-1,2,4,4 $\mathrm{H}$-triazole) for which it was possible to select single crystals with different shapes, on the same batch. Much more than that, it was possible to select a robust single crystal with an irregular shape and to follow the interplay between the interface propagation and the global shape of the crystal. We believe that this synergy between the shape of the HS-LS interface and that of the crystal is a strong indication of the long-range character of the interactions between the SCO molecules that are working within the crystal. Such long-range interactions originate from the local volume change accompanying the spin transition of each molecule and which is delocalized over the other molecules via lattice acoustic phonons. As a consequence, the use of mean-field based models is suitable for the description of the spatiotemporal properties of SCO materials as it will be demonstrated through the manuscript. The manuscript is organized as follows: Section II introduces the experimental technique and the studied crystals, and in Section III, we present our experimental findings on the thermo-induced spin transition of different single crystals and discuss their spatiotemporal features. In Section IV, we establish a comparative study based on a theoretical spatiotemporal model allowing the experimental data to be faithfully described. Section $\mathrm{V}$ concludes and outlines some possible developments of this work.

\section{Experimental details}




\section{Chemical synthesis}

The 2-aminopyridine, diformylhydrazine, potassium tetracyanopalladate $\left(\mathrm{K}\left[\mathrm{Pd}(\mathrm{CN})_{4}\right] \cdot \mathrm{xH}_{2} \mathrm{O}\right)$ and iron(II) perchlorate were purchased respectively from Alfa Aesar and Sigma-Aldrich, and used without further purification. Solvents were used and purified using standard procedures. Elemental analyses were performed by the "Service Central d'Analyses du CNRS", Gif-sur-Yvette, France. Infrared spectra were recorded in the range $4000-400 \mathrm{~cm}^{-1}$ on an FTIR Brucker ATR Vertex 70 Spectrometer. NMR spectra were recorded on a Bruker DRX $300 \mathrm{MHz}$. DSC measurements were performed on a DSC-1/LN2 Mettler Toledo calorimeter setting the heat flow scan rate at $\mathrm{s}=0.4 \mathrm{~K} \mathrm{~min}{ }^{-1}$. The 4-(2-pyridyl)-1,2,4,4 H-triazole (2-pytrz) was prepared using the procedure described in ref. 28.

The compound $\left[\mathrm{Fe}(2-\mathrm{pytrz})_{2}\left\{\mathrm{Pd}(\mathrm{CN})_{4}\right\}\right] \cdot 3 \mathrm{H}_{2} \mathrm{O}(\mathbf{1})$ investigated in the current $\mathrm{OM}$ studies is analogous to $\{\mathrm{Fe}(2-$ pytrz) $\left.\left[\mathrm{Pt}(\mathrm{CN})_{4}\right] \cdot 3 \mathrm{H}_{2} \mathrm{O}\right\}^{29}$ where platinum is replaced by palladium. Single-crystals of 1 were prepared by slow diffusion, in a fine glass tube $(3.0 \mathrm{~mm}$ diameter) of two aqueous solutions: the first solution was obtained by dissolving $\mathrm{K}_{2}\left[\mathrm{Pd}(\mathrm{CN})_{4}\right] \cdot \mathrm{xH}_{2} \mathrm{O}(28.9 \mathrm{mg}, 0.1 \mathrm{mmol})$ in $10 \mathrm{~mL}$. The second solution was prepared by dissolving $\mathrm{Fe}\left(\mathrm{ClO}_{4}\right)_{2} \cdot \mathrm{xH}_{2} \mathrm{O}(25.5 \mathrm{mg}, 0.1 \mathrm{mmol})$ in a solution $(10 \mathrm{~mL})$ of 4 -(2-pyridyl)-1,2,4,4H-triazole ${ }^{28}(29.2 \mathrm{mg}, 0.2 \mathrm{mmol})$. After standing overnight, a light yellow coloration appeared. $2 \mathrm{~mL}$ of the $\mathrm{K}_{2}\left[\mathrm{Pd}(\mathrm{CN})_{4}\right] \cdot \mathrm{xH}_{2} \mathrm{O}$ solution was placed in the fine glass tube and $2 \mathrm{~mL}$ of the yellow solution was added carefully. After three days, colorless small fine square crystals of 1 were formed by slow diffusion. The synthesis of the polycrystalline powder of 1 (yield $65 \%, 199.3 \mathrm{mg}$ ) is detailed in the ESI.t The infrared spectrum of 1 (Fig. S1, ESI $)$ ) shows a strong band at $2170 \mathrm{~cm}^{-1}$ in the cyanide stretching region. The infrared spectrum of the $\mathrm{K}_{2}\left[\mathrm{Pd}(\mathrm{CN})_{4}\right]$ contains a strong band at $2133 \mathrm{~cm}^{-1}$ (Fig. S2, ESI_) which can be attributed to the terminal CN groups. Thus, the strong band observed for 1 can be assigned to a bridging coordination mode of the four $\mathrm{CN}$ groups since bridging $\mathrm{CN}$ ligands typically have higher stretching frequencies than the terminal CN ligands.

\section{Structural properties}

As expected, the structural studies clearly revealed that compound 1 (Fe-Pd) is isostructural to the [Fe(2pytrz) $\left.{ }_{2}\left\{\mathrm{Pt}(\mathrm{CN})_{4}\right\}\right] \cdot 3 \mathrm{H}_{2} \mathrm{O}\left(\mathrm{Fe}-\mathrm{Pt}\right.$ ) parent compound (see Table S1, ESI $\underline{\text { ) }} \cdot{ }^{29}$ Thus as for Fe-Pt, the asymmetric unit of 1 contains two independent Fe(II) ions, Fe1 and Fe2, located on two special positions (an inversion center (1/4 1/4 $1 / 2$ ) for Fe1 and a two-fold axis $(1 / 2$ y $3 / 4)$ for $\mathrm{Fe} 2)$, one $\left[\mathrm{Pd}(\mathrm{CN})_{4}\right]^{2-}$ anion, two 2-pytrz molecules acting as terminal ligands and three water molecules, all located in general positions. As described previously for Fe-Pt, the Fe1 and Fe2 iron centers are alternately linked by the $\left[\mathrm{Pd}(\mathrm{CN})_{4}\right]^{2-}$ anions, acting as tetra-bridging ligands $\left(\mu_{4}-\left[\mathrm{Pd}(\mathrm{CN})_{4}\right]^{2-}\right)$ to form a neutral 2-D coordination polymer of chemical formula [ $\left.\mathrm{Fe}(2-\mathrm{pytrz})_{2}\left\{\mathrm{Pd}(\mathrm{CN})_{4}\right\}\right]$, in which the two 2-pytrz molecules, coordinated to the Fe(II) ions (Fe1 and Fe2), occupy the two axial positions (Fig. 1a). These 2D layers stack along the [ $\left[\begin{array}{ll}1 & 0\end{array}-1\right]$ direction to form a 3-D crystal packing for which the cohesion is ensured essentially by a strong hydrogen-bonding network involving the three water molecules and the uncoordinated nitrogen atom of the triazole group of the 2-pytrz (igi 1 b and Fig. S3, ESI $\underline{\text { }}$ ).

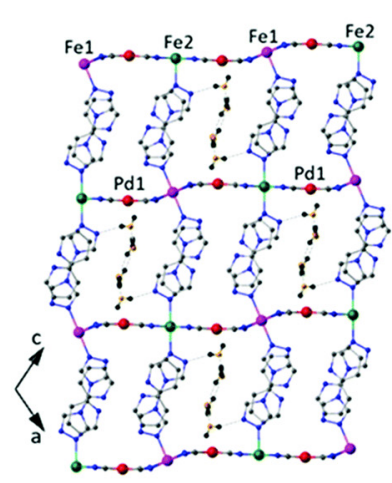

a)

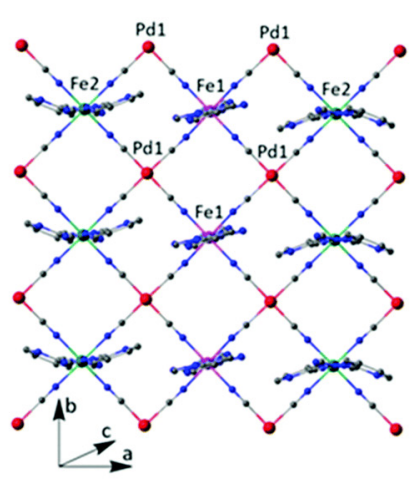

b)

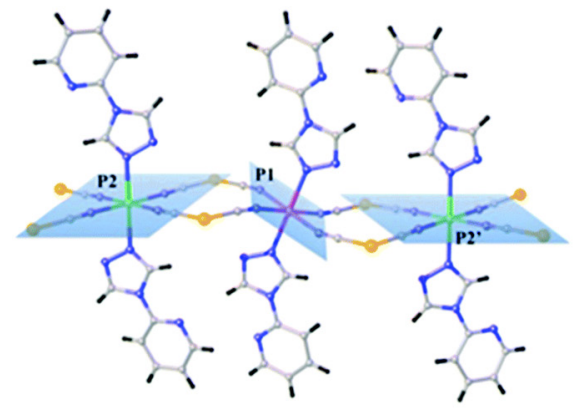

c)

Fig. 1 Structure of $\left[\mathrm{Fe}(2-p y t r z)_{2}\left\{\mathrm{Pd}(\mathrm{CN})_{4}\right\}\right] \cdot 3 \mathrm{H}_{2} \mathrm{O}$ (1) at ambient temperature (298 K): (a) view of the $2 \mathrm{D}$ covalent arrangement of 1; (b) projection view of the 3D crystal packing of 1 in the ac plane showing the two different Fe(II) environments (Fe1 and $\mathrm{Fe} 2$ ) and the hydrogen-bonding network (dashed lines) between Fe2 sites; (c) view along the [010] direction showing the 
$\mathrm{FeN}_{4}$ equatorial plane aligned with the $\left[M(C N)_{4}\right]^{2-}$ plane for Fe2 (P2 and $\left.P 2^{\prime}\right)$ while the Fe1 $N_{4}$ plane $(P 1)$ appears clearly tilted. ${ }^{29}$

As for the $\left[\mathrm{Fe}(2-\text { pytrz })_{2}\left\{\mathrm{Pt}(\mathrm{CN})_{4}\right\}\right] \cdot 3 \mathrm{H}_{2} \mathrm{O}$, the Fe2 environment ( $\mathrm{P} 2$ and $\mathrm{P} 2^{\prime}$, in Fig. $1 \mathrm{C}$ ) which is subjected to the hydrogen-bonding, has a more distorted geometry compared to that of Fe1 (P1, in Fig. 1C) for which the four nonlinear $\mathrm{Fe} 1-\mathrm{N}-\mathrm{C}$ groups cause a significant deviation of the $[\mathrm{Fe} 1(\mathrm{~N} 4)]$ plane from the $\left[\mathrm{Pd}(\mathrm{CN})_{4}\right]^{2-}$ one. As a result, and similar to the parent Fe-Pt compound, only Fe1 exhibits an abrupt spin transition from the HS to LS states, accompanied by a thermal hysteresis loop, while Fe2, experiencing a distorted coordination sphere, remains trapped in the HS state, as shown by the magnetic and DSC studies depicted in Fig. 2a and b, respectively.

a)

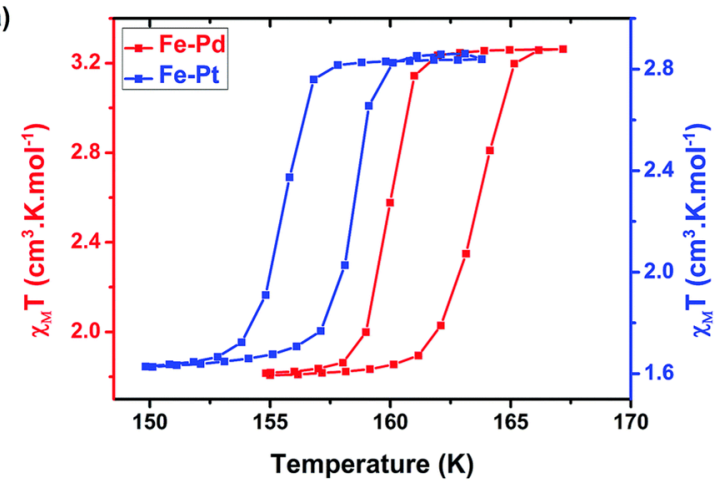

b)

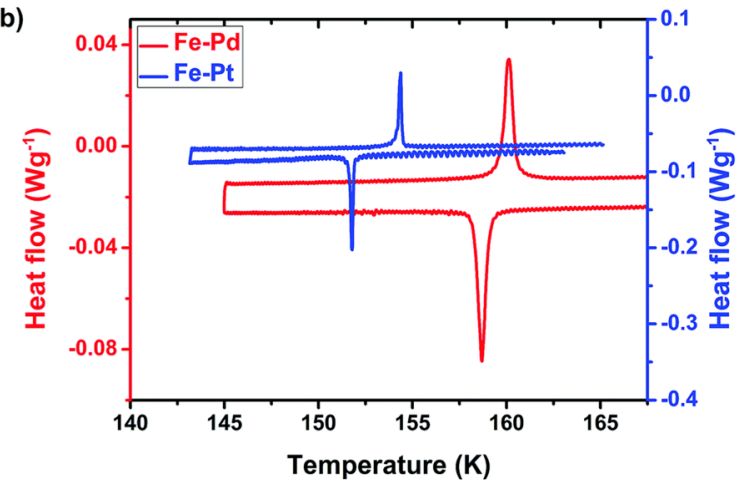

Fig. 2 (a) Thermal variations of the $\chi_{m} T$ products observed for $\left[\mathrm{Fe}(2-\mathrm{pytrz})_{2}\left\{\mathrm{Pd}(\mathrm{CN})_{4}\right\}\right] \cdot 3 \mathrm{H}_{2} \mathrm{O}(\mathbf{1})\left(\mathrm{T}^{\text {down }}{ }_{1 / 2}=160.0 \mathrm{~K}\right.$ and $\mathrm{T}^{\mathrm{up}}{ }_{1 / 2}=$ $163.5 \mathrm{~K})$ and for the parent compound, $\left[\mathrm{Fe}(2-\mathrm{pytrz})_{2}\left\{\mathrm{Pt}(\mathrm{CN})_{4}\right\}\right] \cdot 3 \mathrm{H}_{2} \mathrm{O}\left(\mathrm{T}^{\text {down }}{ }_{1 / 2}=152 \mathrm{~K}\right.$ and $\left.\mathrm{T}^{u p}{ }_{1 / 2}=154 \mathrm{~K}\right){ }^{29}$ (b) DSC studies for 1 (Fe-Pd) and for [Fe(2-pytrz $\left.)_{2}\left\{\mathrm{Pt}(\mathrm{CN})_{4}\right\}\right] \cdot 3 \mathrm{H}_{2} \mathrm{O}$ (Fe-Pt $)^{29}$ showing the exo-and endo-thermic transformations. The upper (resp. lower) transition temperatures, determined from the maximum of the enthalpy peaks, are $160.6 \mathrm{~K}$ (resp. $158.7 \mathrm{~K}$ ) for 1 and

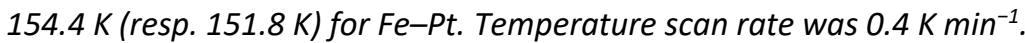

As observed for the $\left[\mathrm{Fe}(2-\mathrm{pytrz})_{2}\left\{\mathrm{Pt}(\mathrm{CN})_{4}\right\}\right] \cdot 3 \mathrm{H}_{2} \mathrm{O},{ }^{29}$ in the plateau region, the spatial distribution of the $\mathrm{HS}$ fraction follows a checkerboard structure made of HS and LS states. This incomplete spin transition induces a half volume change at the transition and is one of the reasons for the resilience of this material. The complete thermal transition requires the material to be switched from the HS-LS intermediate state to the LS-LS state, which can be reached by excitation with red light $(800 \mathrm{~nm})$ at low-temperature, as it will be demonstrated in a forthcoming work.

\section{Magnetic and calorimetric studies}

The magnetic data of Fig. $2 \mathrm{a}$ for 1 , performed at the temperature scan rate of $0.4 \mathrm{~K} \mathrm{~min}^{-1}$, were determined over the 2-300 K temperature range on a polycrystalline sample. The $\chi_{m} T$ value (where $\chi_{m}$ is the magnetic susceptibility and $\mathrm{T}$, the temperature) of $3.22 \mathrm{~cm}^{3} \mathrm{~K} \mathrm{~mol}^{-1}$ at room temperature is consistent with an $\mathrm{HS}(\mathrm{S}=2)$ configuration of the hexa-coordinated Fe(II) ions. At $100 \mathrm{~K}$, the $\chi_{\mathrm{m}} \mathrm{T}$ value of $1.80 \mathrm{~cm}^{3} \mathrm{~K} \mathrm{~mol}^{-1}$ is consistent with an HS-LS configuration (around $57 \%$ of HS residual fraction). The HS-HS to HS-LS transition occurs with a small and abrupt hysteresis loop $\left(\mathrm{T}^{\text {down }} 1 / 2 \sim 160.0 \mathrm{~K}, \mathrm{~T}^{\text {up }}{ }_{1 / 2} \sim 163.5 \mathrm{~K}\right)$.

The bistability of compound $\mathbf{1}$ is fairly confirmed by DSC studies which exhibit extrema of heat flows at 158.7 and $160.6 \mathrm{~K}$, respectively (Fig. 2 b) .

For comparison, we have included in Fig. $2 \mathrm{a}$ and $\mathrm{b}$, the magnetic and DSC data of the parent compound $\{\mathrm{Fe}$ (2pytrz) $\left.\left[\mathrm{Pt}(\mathrm{CN})_{4}\right] \cdot 3 \mathrm{H}_{2} \mathrm{O}\right\}^{29}$ which makes the transitions from LS to HS and $\mathrm{HS}$ to $\mathrm{LS}$ at slightly different temperatures.

\section{Optical microscopy}

Before proceeding to the OM investigations, the first experimental step is the selection of clean single crystals with the minimum number of visible defects on the surface. We have been able to select the two single crystals of Fig. $\underline{3}$, grown in the same batch, with triangular and rectangular shapes, which have typical lengths of $121 \mu \mathrm{m}$ and 136 $\mu \mathrm{m}$. The rectangular and the triangular-shaped single crystals will be abbreviated $\mathrm{R}$ and $\mathrm{T}$ throughout the document. 
For the experimental investigations, we used a Nikon Eclipse LV100 microscope, equipped with an Oxford cryostat and a Dalsa Falcon 1.4M100 camera which can capture up to 100 images per second. The single crystals were placed into a sample cell, which is itself fixed to the cold finger of a helium-flow Oxford cryostat. For efficient thermal control and to ensure thermal homogeneity in the sample cell, we fixed the temperature scan rate at $0.2 \mathrm{~K} \mathrm{~min}^{-1}$ and we configured the camera to save 10 images per second to record the spin transition of the single crystals.

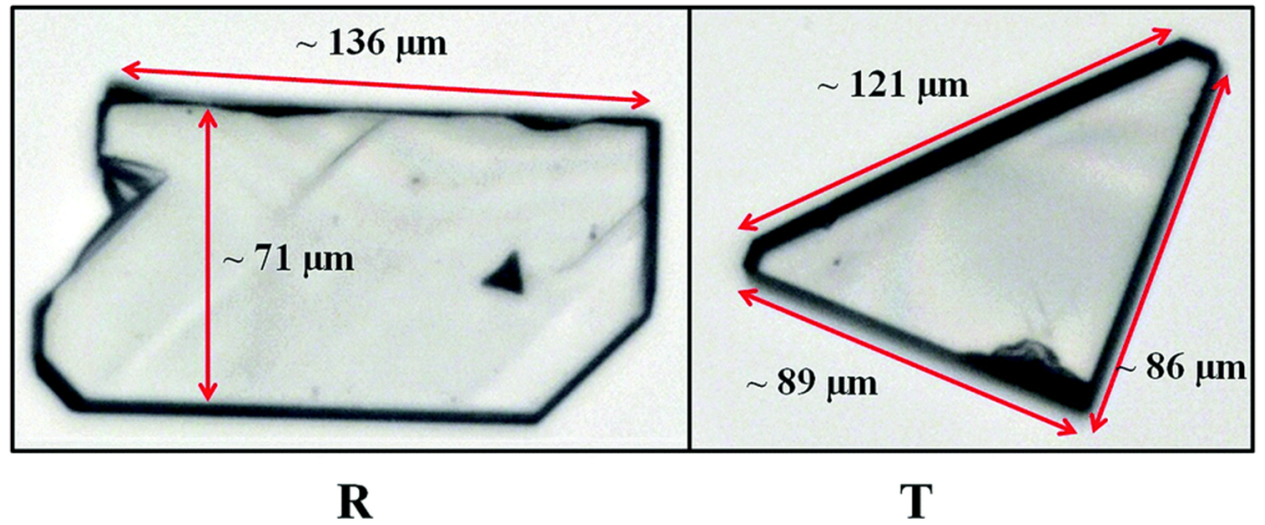

Fig. 3 The rectangular $(R)$ and triangular $(T)$ chosen crystals in the HS state.

\section{Results and discussion}

\section{Thermo-induced spin transition}

The $\left[\mathrm{Fe}(2-\mathrm{pytrz})_{2}\left\{\mathrm{Pd}(\mathrm{CN})_{4}\right\}\right] \cdot 3 \mathrm{H}_{2} \mathrm{O}$ single crystals undergo a first-order phase transition between the LS and $\mathrm{HS}$ states with a hysteresis loop. This thermo-induced spin transition was investigated through the color change of the two selected single crystals using $\mathrm{OM}$ investigations with a spatial resolution of $0.3 \mu \mathrm{m}$. The variation of the transmitted intensity, I, of light through the crystal, relates to the optical density (OD), which is defined as the decimal logarithm of the ratio of incident/transmitted intensities,

$$
\mathrm{OD}=\log _{10} \frac{I_{0}}{I}
$$

In the transmission geometry, $I_{0}$ is merely the bright field intensity, and I can be determined, either locally, or on average over a defined area of the crystal. For the determination of the HS fraction, we only consider average values, so as to reach the high signal/noise ratio values required for a detailed analysis of the temporal aspects of the phase transformations. The OM data (the recorded images) were treated using Matlab programs developed in the Versailles group. ${ }^{30}$ For qualitative investigations, each image was split into three OD components: red, green and blue. The minimum noise data were obtained with the green OD. Since the transmitted intensity depends on the crystal thickness, and so the $O D$, it is then difficult to use this quantity as an absolute parameter for comparing the rate of HS fraction from one crystal to another. In contrast, the normalized OD, defined as

$$
n=\frac{\mathrm{OD}(x, y, t)-\mathrm{OD}(\mathrm{HS})}{\mathrm{OD}(\mathrm{HS})-\mathrm{OD}(\mathrm{LS})}
$$

(where $\mathrm{OD}(\mathrm{HS})$ and $\mathrm{OD}(\mathrm{LS})$ are respectively the $\mathrm{OD}$ values in the HS and LS states) directly relates to the HS fraction, $\mathrm{n}_{\mathrm{HS}}$, during the transition, which is the common order parameter used in SCO materials.

In Fig. 4, we reported the hysteresis loops of $\mathrm{R}$ and $\mathrm{T}$ single crystals, derived from monitoring the OD during the spin transition. The corresponding transition temperatures were found to be very close to each other: $T_{\text {up }}=163 \mathrm{~K}$ and $\mathrm{T}_{\text {down }}=152.8 \mathrm{~K}$ for $\mathrm{R}$, and $\mathrm{T}_{\text {up }}=162 \mathrm{~K}$ and $\mathrm{T}_{\text {down }}=150.43 \mathrm{~K}$ for $\mathrm{T}$, while both magnetic and DSC studies performed on 
a set of single crystals of different sizes and shapes led to $T_{\text {up }}=163.5$ and $160.6 \mathrm{~K}$ and $T_{\text {down }}=160.0$ and $158.7 \mathrm{~K}$, respectively. These values are summarized in Table 1, where we have also reported the approximated equilibrium transition temperatures,

$$
T_{\text {eq }} \sim \frac{T_{\text {up }}+T_{\text {down }}}{2}
$$

and the thermal hysteresis widths, $\Delta T$, for the two single crystals, $R$ and $T$. One can easily notice that $T_{\text {eq }}$ and $\Delta T$ are in very good agreement with the magnetic data of a powder sample, whose corresponding hysteresis loop is reported in the inset of Fig. 4. The slight difference in the transition temperatures between OM on the one hand and the magnetic and DSC data on the other, is attributed to the fact that magnetic and DSC measurements result in the statistical average of distributed transition temperatures of a large number of single-crystals with different shapes and sizes, whereas OM data relate to one single crystal's behavior.

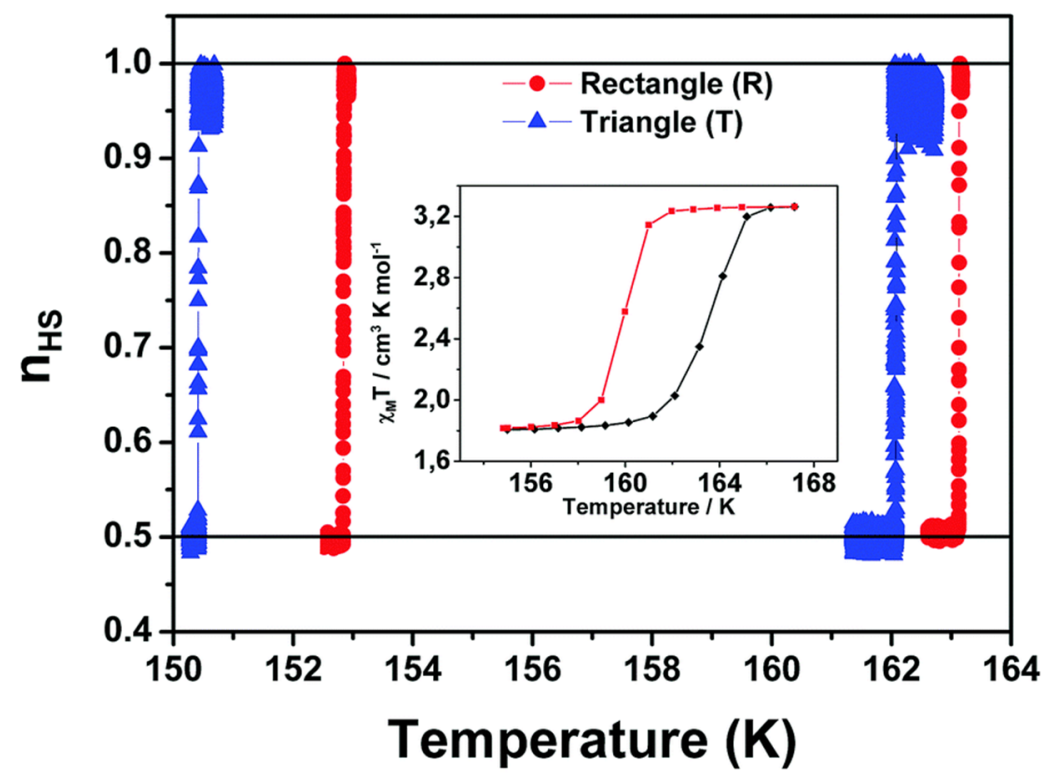

Fig. 4 Optical microscopy and magnetic (in inset) thermal hysteresis loops. For OM, the hysteresis of a $T$ (resp. R) single crystal is shown with blue (resp. red) dots. The temperature rate was $0.2 \mathrm{~K} \mathrm{~min}^{-1}$, on both cooling and heating processes for OM measurements and $0.4 \mathrm{~K} \mathrm{~min}^{-1}$ for the magnetic data.

Table 1 Switching temperatures and hysteresis widths of single crystals of 1 using the OM, magnetic and DSC studies

\begin{tabular}{lclll}
\hline Crystal & $\begin{array}{l}\text { Rectangle } \\
(\mathrm{OM})\end{array}$ & $\begin{array}{l}\text { Triangle } \\
(\mathrm{OM})\end{array}$ & $\begin{array}{l}\text { Magnetic } \\
\text { studies }\end{array}$ & $\begin{array}{l}\text { DSC } \\
\text { studies }\end{array}$ \\
\hline$T_{\text {down }}(\mathrm{K})$ & 152.8 & $\mathbf{1 5 0 . 4}$ & 160.0 & 158.7 \\
$T_{\text {up }}(\mathrm{K})$ & 163 & 162 & 163.5 & 160.6 \\
Equilibrium temperature $(\mathrm{K})$ & 157.9 & $\mathbf{1 5 6 . 2}$ & 161.75 & 159.65 \\
Hysteresis width (K) & 10.2 & 11.5 & 3.5 & 1.9
\end{tabular}

\section{Spatiotemporal properties of the thermo-induced spin transition}

At this stage, we should mention that our single crystals are very robust compared to some prior studies by optical microscopy where the crystals underwent considerable mechanical stresses leading to fractures and cleavages. ${ }^{20,21}$ Both single crystals R and T of Fig. 3, exhibit nucleation and growth processes during their thermo-induced spin transition, for which we observed well-defined interfaces between the two spin states (HS-HS and HS-LS) of the two Fe(II) centers (Fe1 and Fe2) that spread over the whole single crystals. Fig. 5a displays a set of selected snapshots of the single crystals during their thermo-induced spin transition. We remark that for both single crystals, 
the new phase appears from the most acute angle (see Fig. 5a and movies SM1-SM4 of the ESII). For example, in the rectangular crystal, the stable phase nucleates from the right corner (the most acute angle) for both cooling and heating processes. However, in the triangular crystal there are two corners with approximately the same acute angle $\left(\sim 49^{\circ}\right)$, and thus the probabilities of nucleation from both corners are almost identical. As a result, the nucleation started from one corner on cooling and the other on heating, as confirmed by the snapshots of Fig. $5 \mathrm{a}$.

\section{Propagation velocity of the HS-LS interface}

By tracking the spatiotemporal evolution of the green OD evaluated in each pixel ( 1 pixel $\sim 0.3 \times 0.3 \mu \mathrm{m}^{2}$ ), using a specific Matlab program designed for the treatment and image processing of OM data, ${ }^{30}$ we have been able to monitor the interface position as a function of time, as illustrated in Fig. $5 \mathrm{~b}$ and c. We noticed, in both single crystals, that the velocity of the HS-LS interface in the cooling process is higher than that on heating. The average velocities are summarized in Table 2. A meticulous inspection of the time evolution of the interface's position reveals the existence of different regimes of velocity, related to the irregular macroscopic shapes of the single crystals, in particular in crystal R. Indeed, one can easily see in the snapshots of Fig. 5a that the interface changes its orientation and shape during the propagation so as to fit the system's borders and corners. In other words, the HS-LS interface, whose origin arises from the difference of lattice parameters between the LS and HS phases, optimizes its shape and length so as to minimize the elastic energy of lattice mismatch stored in its width. ${ }^{22,24,25}$ Accordingly, it is expected that the velocity of the interface changes during the propagation process. Such a fact is remarkable when the interface approaches the extremity of the single crystal where it accelerates in such a way that the velocity was multiplied by a factor of 5 . Such acceleration is in good agreement with previous theoretical studies $^{24,31}$ based on elastic description of the SCO phenomenon, taking into account the volume change at the transition. ${ }^{31}$

a)

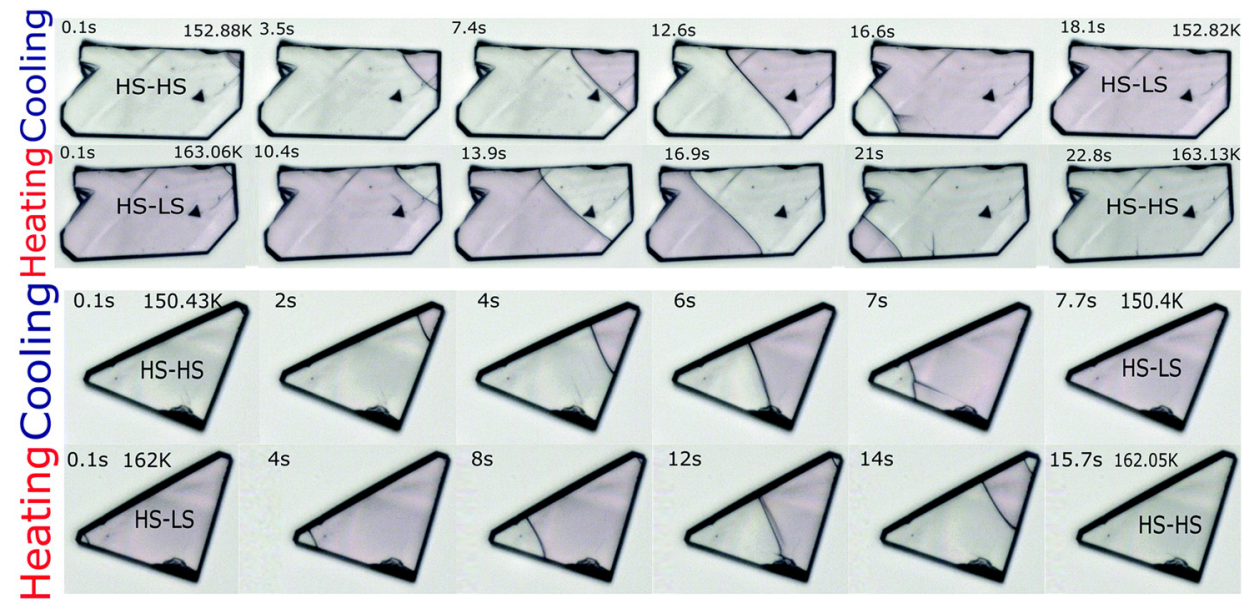

b)

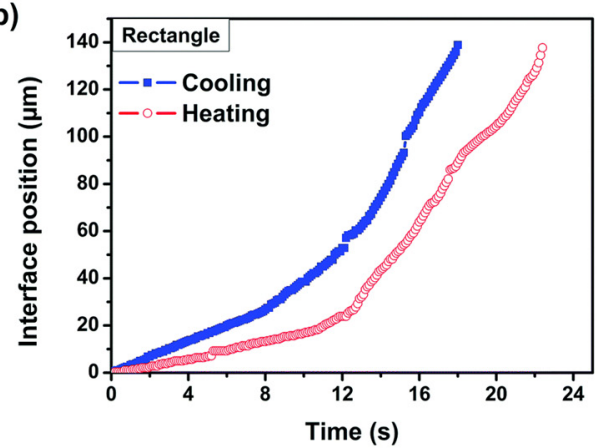

c)

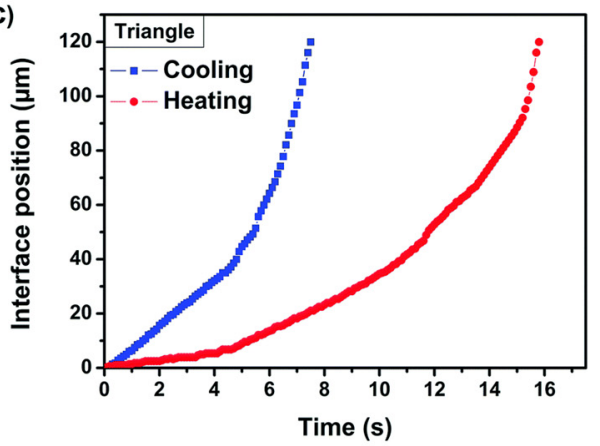

Fig. 5 Snapshots of rectangular-and triangular-shaped crystals during their thermo-induced spin transition on cooling and heating (a). The interface position as a function of time along the direction of propagation in $R(b)$ and $T(c)$ single crystals.

The interface position was estimated on a straight line perpendicular to the interface. Notice the existence of several regimes of propagation directly related to the macroscopic shapes of the crystals. The average velocities of front propagation are given in Table 2. 
Table 2 Average interface velocities in the linear regime of the propagation for cooling and heating processes of the two single crystals

\begin{tabular}{lll}
\hline Crystal & Rectangle & Triangle \\
\hline Interface velocity on cooling $\left(\mu \mathrm{m} \mathrm{s}^{-1}\right)$ & 4.4 & 8.5 \\
Interface velocity on heating $\left(\mu \mathrm{m} \mathrm{s}^{-1}\right)$ & 2.5 & 5.5
\end{tabular}

The quantitative time-dependence of the interface positions for $\mathrm{R}$ and $\mathrm{T}$ crystals are presented in Fig. $5 \mathrm{~b}$ and $\mathrm{c}$. As we have mentioned before, they clearly allow the identification of several regimes of propagation velocities, attributed to the change of the interface shape. So, it becomes mandatory to investigate the local velocity of each interface point during its propagation. Before going further, it is worth noticing that the whole transformation process takes roughly,

$$
\Delta t=\frac{L}{v} \sim 10(\text { resp. 20) }
$$

, seconds during cooling (resp. heating), where $L$ is the crystal size and $v$ is the average interface velocity (given in Table 2). From the knowledge of the temperature scan rate, $r=0.2 \mathrm{~K} \mathrm{~min}^{-1}$, we evaluated the crystal temperature variation along the heating branch as only $0.17 \mathrm{~K}$ at the maximum. This value is very small compared to the width $(\sim 10 \mathrm{~K})$ of the thermal hysteresis, which clearly points-out that the transformation process is almost "isothermal" and very sharp, thereby confirming the first-order character of this cooperative transformation.

\section{The velocity field.}

We performed in Fig. 6 , the mapping of the positions of the HS-LS interfaces on the sample, on which we reported the field of local interface velocities, calculated as

$$
\vec{v}(x, y)=\frac{\Delta s}{\Delta t} \frac{\vec{\nabla} n_{\mathrm{HS}}}{\left\|\vec{\nabla} n_{\mathrm{HS}}\right\|},
$$

where $\vec{\nabla} n_{\mathrm{HS}}$, is the gradient of the HS fraction which gives the local direction of the interface velocity and $\Delta \mathrm{s}$ is the local interface displacement (along the $\vec{\nabla} n_{\mathrm{HS}}$ direction) during the time interval, $\Delta$ t. The gradient of the HS fraction relates here to that of the OD at the considered point, which is derived from OM data. Fig. 6a illustrates the map of the field velocities of the interface on the crystal $R$ in the cooling process and Fig. $6 \mathrm{~b}$ illustrates that of crystal $\mathrm{T}$, on heating. In both figures, the time interval between two successive interfaces is 1 second. First, we remark that the global behavior of nucleation and growth is quasi similar for both single crystals, and in most of the cases, the interface is more or less perpendicular to the edge of the crystal, except in the beginning of the process. We can also notice that the amplitude of the local interface velocity vectors plotted in Fig. 6a and b differs from one interface to another and also along the same interface. This clearly proves the self-adaptation of the interface shape to the boundary conditions imposed by (i) the global shape of the crystal and (ii) the eventual presence of defects on the way of the interface path. For both crystals, we noticed that the interface velocity is very slow at the beginning of the transition process, which suggests the existence of an incubation regime at early stages of the thermal transition process. Furthermore, the interface accelerates in the course of the transformation, and its velocity reaches a maximum value at the end of the process, nearby the crystal's frontier. This last fast regime of propagation is attributed to an efficient release of the elastic stress (largely stored in the interface region) in the vicinity of the crystal's surface, thus confirming the predictions of the theoretical studies. ${ }^{25}$ 

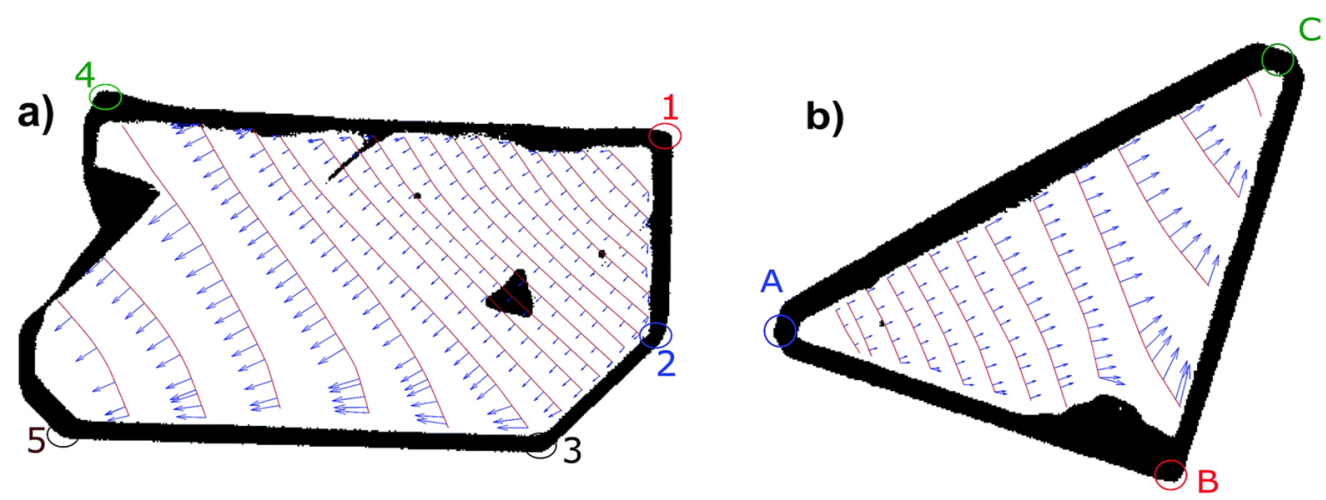

Fig. 6 (a) The experimental field of velocities superimposed over the interface lines (in red) for the rectangular crystal on a cooling process; (b) the triangular crystal during the heating process. The time interval between two interfaces is $1 \mathrm{~s}$ for both single crystals.

To quantify the interplay between the interface and the crystal's shape, we followed the change of the interface speed along the bottom edge in the R crystal (see Fig. 6a), in the cooling regime, starting from point 1 and following the sequence $1 \rightarrow 2 \rightarrow 3 \rightarrow 4 \rightarrow 5$. The evolution of the interface velocity along this line as a function of temperature, displayed in Fig. 7a, clearly reveals four well identified velocity propagation regimes, changing exactly at specific points that correspond to the shape change in the crystal. Interestingly, when the interface meets the crystal asperities, we observe a self-adaptation of its shape, velocity, length and orientation. Consequently, these singular points can be viewed as points at which the border conditions are suddenly changed. The detailed analysis shows that in the first regime $1 \rightarrow 2$, where the crystal shape is regular, the interface keeps the same shape and its velocity along the border is quasi constant and very low. When reaching point 2 , where the interface meets the first singularity, its velocity suddenly increases. A new regime $2 \rightarrow 3$ starts, where the contact angle between the interface and the crystal border is almost $\pi / 2$. The next singularity is encountered at point 3 , from which the top and bottom borders of the crystal become parallel. In this regime $3 \rightarrow 4$, the interface shape distorts and becomes sigmoidal because its upper point (in contact with the upper border of the crystal) is ahead of the lower one. An inspection of the average speeds of the interface points in this region shows that, the lower point accelerates (Fig. 7a) and its velocity reaches the highest value, $22 \mu \mathrm{m} \mathrm{s}^{-1}$, at point 4 . Concomitantly, the interface straightens slowly during its motion, as illustrated in Fig. $7 \mathrm{~b}$ by the decrease of the distance, $\Delta \mathrm{L}$, (along the propagation direction) between the upper and lower points. This behavior is interesting because it underlines the tendency of the interface to minimize its length while respecting the constraints of boundary conditions, imposed by the contact angles with the crystal edges. It is worth noticing that this behaviour meets the theoretical results of interface propagation in a rectangular-shaped crystal, based on elastic descriptions, ${ }^{23,32,33}$ of the SCO phenomenon, which showed that for an isotropic transformation of the unit cell between LS and HS, the interface is perpendicular to the length of the crystal. $^{34,35}$ Indeed, this configuration corresponds to the shortest interface length, which is the one which minimizes the elastic energy resulting from the lattice parameter misfit between the two phases. Moreover, prior theoretical investigations on the spatiotemporal aspects of the spin transition, based on Monte-Carlo simulations, showed that the geometry of the single crystal controls the interface dynamics on the one hand, and the elastic energy is strongly correlated with the length of the interface, ${ }^{24,25,36,37}$ on the other. This reasoning has however severe limitations, when the change in the unit cell between the LS and HS is anisotropic, as we have reported in the single crystal $\left[\left\{\mathrm{Fe}(\mathrm{NCSe})(\mathrm{py})_{2}\right\}_{2}\left(\mu\right.\right.$-bpypz)]. ${ }^{22,26,27}$ There, although the crystal has a rectangular shape, the interface is found to be tilted by $60^{\circ}$ with respect to the crystal length. 

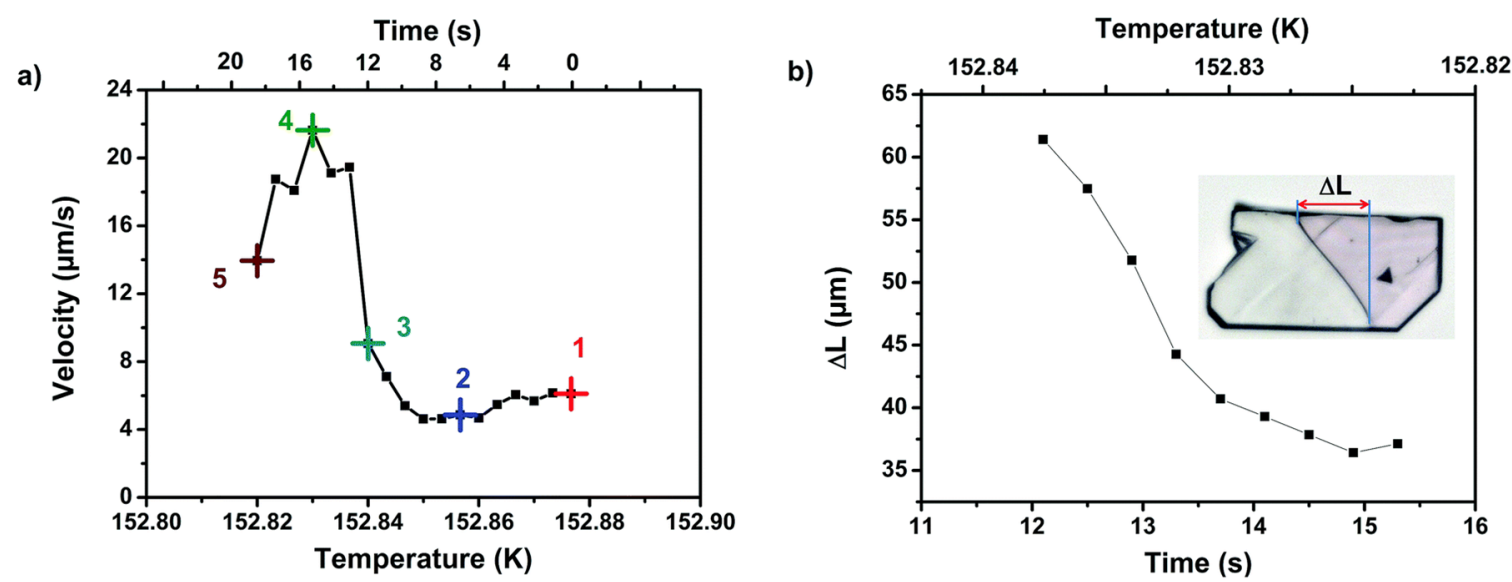

Fig. 7 (a) Temperature-dependence of the interface's velocity along the pathway $1 \rightarrow 2 \rightarrow 3 \rightarrow 4 \rightarrow 5$ of Fig. $6 a$, showing the existence of four propagation regimes, directly related to the shape of the crystal. See text for more explanations. (b) Temporal evolution of the shift $\Delta L$ between the top and the bottom extremities of the interface (in $3 \rightarrow 4$ region). Inset shows $\Delta L$, represented on the crystal's image.

For the present study, according to the velocity at which the shift $\Delta$ L vanishes with time in the regime $3 \rightarrow 4$, we predict that for a longer and regular crystal, one should obtain a straight interface after $3.5 \mathrm{~s}$ of the propagation process. Since the whole interface moves with an average velocity of $\sim 16 \mu \mathrm{m} \mathrm{s}^{-1}$, and if we admit that this velocity remains constant for bigger crystals, then for observing a straight interface one needs a crystal of $\sim 190 \mu \mathrm{m}$ length instead of the $\sim 136 \mu \mathrm{m}$ of that of Fig. $6 \mathrm{a}$. When reaching the corner 4 , the interface bends, then the last regime 4 $\rightarrow 5$ takes place, where the HS/LS interface meets a series of asperities related to the irregular shape of the crystal's border, resulting in the slowing down of the interface propagation, as clearly depicted in Fig. 7a.

The same procedure was followed for the T single crystal (Fig. 6b) for which the nucleation of the stable HS phase started from the bottom corner A with a curved interface propagating with a perpendicular contact angle with the long edge of the triangle. Here again, we see that the interface curvature changes significantly when it reaches point $B$ with a clear increase of the intensity of the field velocity in the regime $B \rightarrow C$. The comparison between the behaviours of $R$ and $T$ single crystals shows that their interface shapes emerge for their interplay with the crystal borders. This leads to very different average velocities of propagation (Table 2), since whether in the heating or cooling regimes, the interface speed in $\mathrm{T}$ is almost two times greater than that in the $\mathrm{R}$ single crystal.

\section{Interface length during the propagation process}

Now, we focus on the crystal shape dependence of the HS-LS interface length during the propagation process. Although this effect was observed on both R and T single crystals, we will discuss here only the case of the R crystal. Along the growth process, the single crystals change their volumes and generate long-range mechanical stresses that drive the interface propagation. Because of the long-range nature of the elastic interactions induced by the volume change, the geometry of single crystals constitutes an important parameter which influences the shape and the length of the interface..$^{22-25,38}$

In Fig. 8a, we monitored, during the cooling process, the time-dependence of the interface's length, $L$, as well as the fraction of the low-temperature HS-LS $(\mathrm{HL})$ phase, derived from the ratio of phase area,

$$
n_{\mathrm{HL}}=\frac{S_{\mathrm{HL}}}{S_{\mathrm{tot}}}
$$

, in the $\mathrm{R}$ crystal, where $\mathrm{S}_{\mathrm{HL}}\left(\mathrm{S}_{\text {tot }}\right)$ is the area of the $\mathrm{HL}$-phase (crystal). The fraction, $\mathrm{n}_{\mathrm{HL}}$, is an increasing function with time, following a sigmoidal process, which supports the cooperative character of the present SCO single crystals. On the other hand, the length of the interface is strongly connected to the amount of elastic energy convoyed during the interface propagation. Considering a negligible change in the interface width during the process, one can expect a linear relationship between the interface's length and the HL fraction. Fig. $8 \mathrm{~b}$ shows that 
this correlation is verified inside each regime prior to any incident in the interface propagation due to the irregularities of the crystal shape.
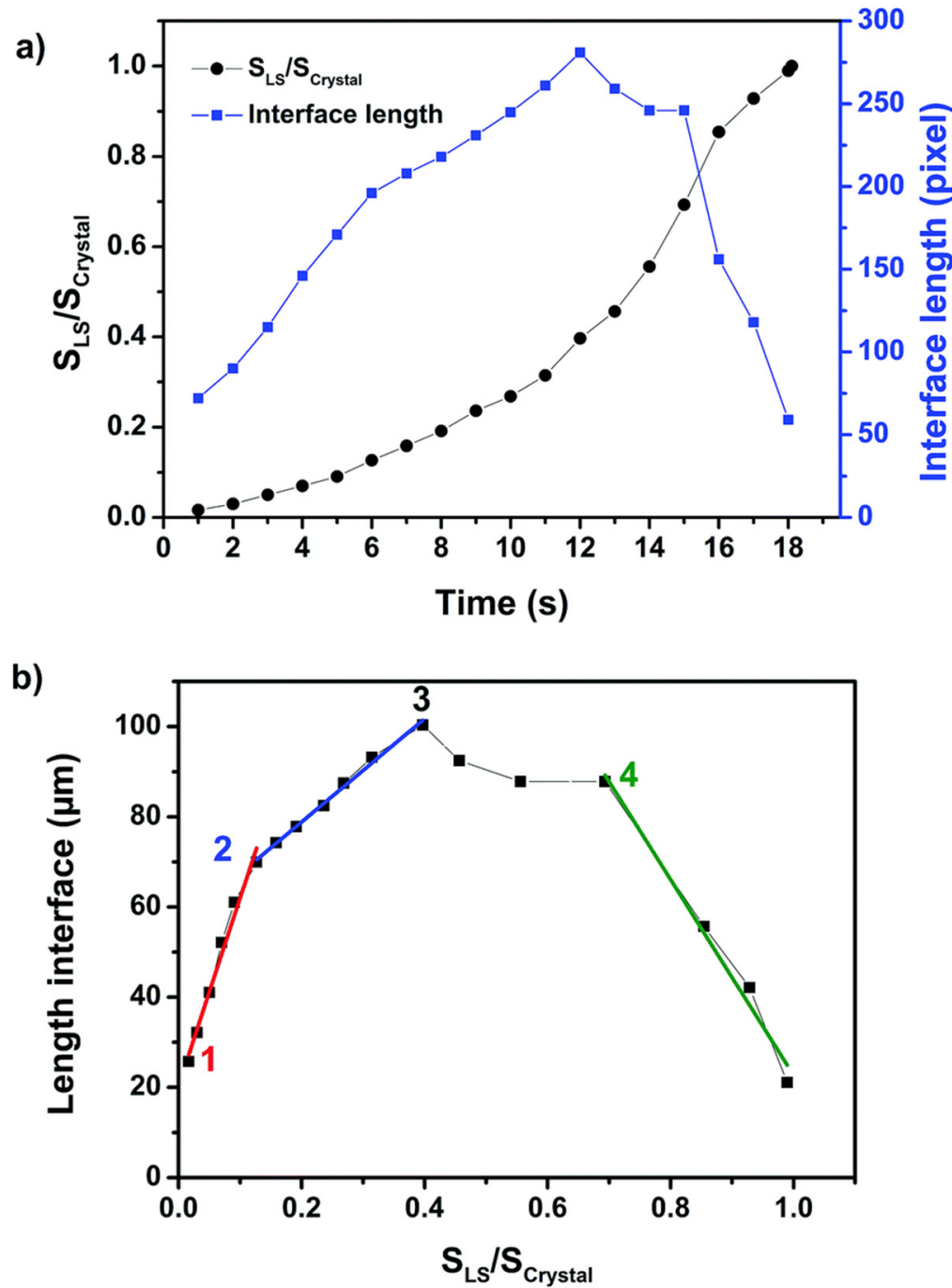

Fig. 8 (a) Experimental temporal evolution of the interface length (blue line) and HS-LS fraction, $n_{H L}=S_{L S} / S_{\text {tot }}(b / a c k$ line). (b) Correlation graph giving the interface length vs. $n_{H L}$. An excellent linear correlation is obtained in all regimes, except for the third one.

In summary, we identified four velocity regimes in the propagation process of the rectangular crystal: three are linear and one is non-linear. All of them were correlated with the border conditions as illustrated in Fig. 8b. The first one (denoted by a red line) corresponds to the beginning of the nucleation process in the top right acute corner of the crystal (point 1 in Fig. 6a), from which the HS-LS domain emerges with a curved interface having roughly right contact angles (regime $1 \rightarrow 2$ ). The second one (blue line) is triggered by the angle change at point 2 , which affects the boundary conditions, inducing a new shape of the interface. From point 3 , the interface has a sigmoidal shape and its propagation is a bit disturbed with visible defects as could be seen in Fig. 6a. Finally, in the last linear regime $4 \rightarrow 5$ (green line in Fig. 8 b), the circular shape of the interface is restored.

\section{Comparison between OM and X-ray diffraction data}

OM was used to perform a quantitative analysis of the change of the crystal size between the LS and the HS states. This analysis was performed on both R and T crystals at 140 and $180 \mathrm{~K}$, corresponding to LS and HS states, respectively. The images of the crystals at these temperatures are displayed in Fig. S4 of the ESI. \pm We found that crystal R undergoes an elongation of 1.4\% (1.6\%) along the length between points 1 and 4 ( 3 and 5 ) of Fig. 6 upon LS to HS transition. Similarly, an expansion of $2.2 \%$ was evaluated along the width between points 1 and 2 . On the other hand, crystal $T$ exhibited an expansion of $1.8 \%$ between points $A$ and $B$ and $2.2 \%$ between points $B$ and $C$. 
Thus, the data of the T crystal coincide with those of the R crystal, which clearly proves that crystal $T$ is not the result of polymorphic modifications. Overall, these values are in excellent agreement with X-ray data which indicated an elongation of $2.17 \%$ of the a parameter and $1.8 \%$ of the $b$ parameter, while that of the $c$ parameter is only $0.6 \%$. These data are summarized in Table 3 , which allow it to be identified that the length and the width of crystal R correspond to crystallographic directions, $b$ and $a$. In light of the above we conclude that the front propagation takes place in the $a-b$ plane of this 2-D coordination polymer, where the most important changes in lattice parameters are observed at the transition.

Table 3 Comparison of the relative variation of crystal sizes derived from OM data and those of the unit cell parameters $a, b$, and $c$ obtained from $\mathrm{X}$-ray measurements on the isostructural compound [Fe(2-pytrz $\left.)_{2}\left\{\mathrm{Pt}(\mathrm{CN})_{4}\right\}\right]$. $3 \mathrm{H}_{2} \mathrm{O}^{29}$ between HS-HS and HS-LS states. The OM data are derived from Fig. S4, displayed in the ESI

\begin{tabular}{lcll}
\hline X-rays data & & & \\
\hline Lattice & HS-HS & HS-LS & Relative \\
parameter $(\AA)$ & $(296 \mathrm{~K})$ & $(120 \mathrm{~K})$ & variation $(\%)$ \\
\hline$a$ & 25.248 & 24.795 & 1.82 \\
$b$ & 7.4044 & 7.247 & 2.17 \\
$c$ & 27.293 & 27.105 & 0.69 \\
\hline OM data & & & \\
\hline & HS-HS & HS-LS & Relative \\
Crystal R $(\mu \mathrm{m})$ & $(180 \mathrm{~K})$ & $(140 \mathrm{~K})$ & variation $(\%)$ \\
\hline$d_{1-4}$ & 136.8 & 134.9 & 1.4 \\
$d_{1-2}$ & 46.5 & 45.4 & 2.2 \\
$d_{3-5}$ & 115.3 & 113.4 & 1.6 \\
\hline OM data & & & \\
\hline & HS-HS & HS-LS & Relative \\
Crystal T $(\mu \mathrm{m})$ & $(180 \mathrm{~K})$ & $(140 \mathrm{~K})$ & variation $(\%)$ \\
\hline$d_{\mathrm{A}-\mathrm{B}}$ & 89 & 87.4 & 1.8 \\
$d_{\mathrm{B}-\mathrm{C}}$ & 86.6 & 84.7 & 2.2 \\
& & &
\end{tabular}

\section{Theoretical description}

Before we start discussing the model developed for the analysis of the spatiotemporal properties of SCO single crystals, we first briefly introduce the necessary prerequisite related to the theoretical description of thermodynamic properties of SCO materials. A simple model allowing the SCO phenomenon to be accurately mimicked is based on the description of the SCO molecule by a two-states fictitious spin, s, whose eigenvalues -1 and +1 are respectively associated with the LS and the HS states. Due to the difference of the spin states of the molecule between the $\mathrm{LS}(\mathrm{S}=0)$ and $\mathrm{HS}(\mathrm{S}=2)$ states, and the volume expansion in the HS state, the vibrational properties of the lattice are significantly changed, and it results that the HS state is softer than the LS state. The combined increase of volume and spin state in the HS phase compared to the LS state, confers to the former a higher effective degeneracy, $\mathrm{g}_{\mathrm{HS}} \gg \mathrm{g}_{\mathrm{LS}}$. At $0 \mathrm{~K}$, the LS is stabilized by the ligand field energy, $\Delta$, arising from the close nitrogen atoms surrounding the Fe(II) metal. The SCO transition could be thus described using the wellknown Ising-like, ${ }^{38-40}$ model, whose Hamiltonian writes

$$
H=-J \sum_{i} s_{i} s_{j}+\Delta_{\mathrm{eff}} \sum_{i} s_{i}
$$

where $(J>0)$ stands for the ferroelastic interaction between the spin states and $\Delta_{\text {eff }}=\Delta-k_{B} T \ln g$ is the effective energy gap, which contains the contributions of the ligand field energy, $\Delta$, and the degeneracies ratio, $g=g_{\mathrm{HS}} / \mathrm{g}_{\mathrm{LS}}$, between the LS and HS states, which enters under the form of an entropic term in the effective ligand-field energy. The mean-field analysis of Hamiltonian (2) leads quite easily to the following homogeneous, free energy: 


$$
F_{\text {hom }}=\frac{1}{2} J m^{2}-k_{\mathrm{B}} T \ln \left[\left(2 g \cosh \frac{J m-\Delta_{\text {eff }}}{k_{\mathrm{B}} T}\right)\right] \text {, }
$$

Where $m=\langle s\rangle$, is the average fictitious magnetization per site. From the analytical expression of the free energy, given in eqn (3) one can derive straightforwardly the self-consistent equation, through the relation, $\frac{\partial F_{\text {hom }}}{\partial m}=0$,

$$
m=\tanh \beta\left[J m-\Delta_{\mathrm{eff}}\right]
$$

Where $\beta=\frac{1}{k_{\mathrm{B}} T}$.

The HS fraction $\left(n_{H S}\right)$, which is the fraction of molecules occupying the HS state, is simply related to the fictitious magnetization as

$$
n_{\mathrm{HS}}=\frac{1+m}{2}
$$

A brief look at eqn (4) shows that $m=0$ is always a solution when $\Delta_{\text {eff }}=-\Delta \mathrm{k}_{\mathrm{B}} \mathrm{T}$ In $\mathrm{g}=0$. Once $\mathrm{m}=0$ thus ${ }^{n_{\mathrm{HS}}}=\frac{1}{2}$, this means that the temperature cancelling the effective field is the transition temperature of the system, the expression of which writes, $T_{\mathrm{eq}}=\frac{\Delta}{k_{\mathrm{B}} \ln g}$.

Without going into further detail, we mention that the phase diagram of the present model is very simple: (i) a firstorder spin transition with a thermal hysteresis is obtained when $J>k_{B} T_{e q}$ and (ii) a gradual spin conversion takes place otherwise.

In Fig. 9, we depict the case of a thermally-induced first-order spin transition obtained with the following model parameters: $\Delta=394 \mathrm{~K}, \mathrm{~g}=1097$, and $\mathrm{J}=155 \mathrm{~K}$, given in units of temperature $\left(\mathrm{k}_{\mathrm{B}}=1\right)$. The associated thermal hysteresis consists of three main regions, that are represented by a black line for the stable one, a red line for the metastable state and a blue one for the unstable region, the latter being easily identified using the dependence of the free energy (eqn (3)) on the order parameter, $m$.

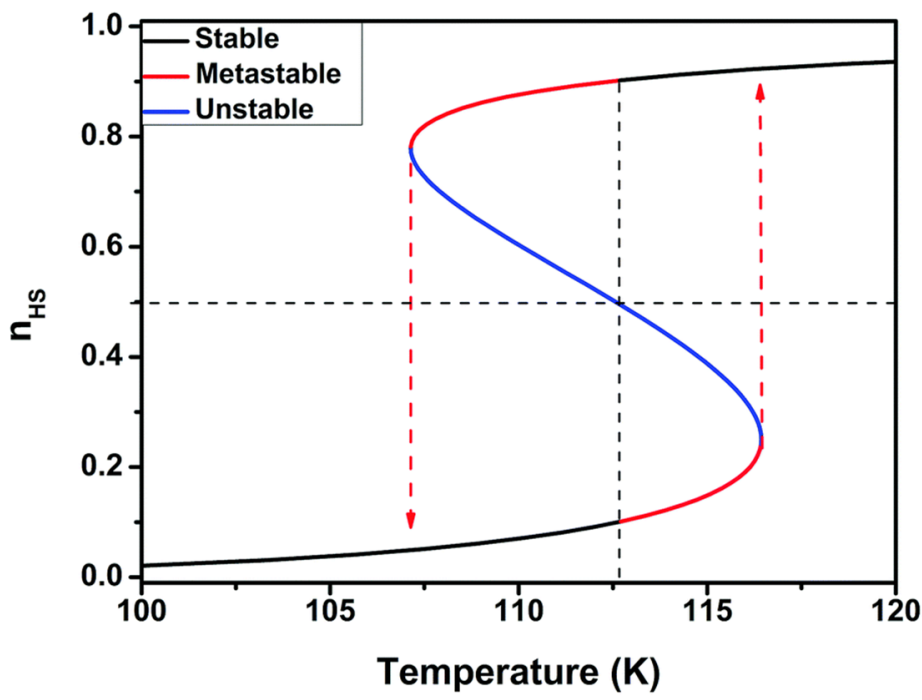

Fig. 9 Thermal-dependence of the HS fraction showing a hysteresis loop with respective upper and lower transition temperatures of 116 and $106 \mathrm{~K}$. The parameter values are, $\Delta=394 \mathrm{~K}, \mathrm{~g}=1097, \mathrm{~J}=155 \mathrm{~K}$. 
The presented experimental spatiotemporal results derived from OM studies on the SCO single crystal, [Fe(2pytrz $\left.{ }_{2}\left\{\mathrm{Pd}(\mathrm{CN})_{4}\right\}\right] \cdot 3 \mathrm{H}_{2} \mathrm{O}$, are interpreted here using a reaction diffusion model, developed in the group ${ }^{36,38}$ which takes into account the local change in the HS fraction, and its spatial variation along the spin transition process. In contrast with the previous homogeneous mean-field approach where the HS fraction (or the magnetization) only depended on temperature, here we extend this study to include time and space in the order parameter. Based on a standard expansion of the mean-field energy (eqn (2)) as a function of the gradient of the "magnetization", $m$, we can establish, as reported in a prior work, ${ }^{38}$ that the spatiotemporal properties of the SCO phenomenon can be described by the following reaction-diffusion equations:

$$
\begin{gathered}
\frac{\partial m}{\partial t}=\Gamma J\left[-m+\tanh \beta\left(J m-\Delta_{\text {eff }}\right)\right]+\vec{\nabla}^{T} \times D \times \vec{\nabla} m \\
\frac{\partial T}{\partial t}=r
\end{gathered}
$$

where the left-side term in eqn (6) is the time derivative of the magnetization, and on the right-side we recognize in the first term, the reaction term related to the driving force $-\frac{\mathrm{d} F}{\mathrm{~d} m}$ (where $\mathrm{F}$ is the mean-field free energy), and the second term is the effective diffusion of the spin state, which emerges from the expansion of $F$ as a function of $\vec{\nabla} m$. Here, for reasons of generality, the diffusion term is written with a tensor diffusion coefficient, which accounts for the possible existence of anisotropic effects, which may arise from the structure of the material. When developed, in the Cartesian basis, this contribution gives the following eqn (8),

$$
\begin{aligned}
\vec{\nabla}^{T} \times D \times \vec{\nabla} m & =\vec{\nabla}^{T} \times\left(\begin{array}{cc}
D_{x x} & D_{x y} \\
D_{y x} & D_{y y}
\end{array}\right) \times \vec{\nabla} m \\
& =D_{x x} \frac{\partial^{2} m}{\partial x^{2}}+D_{y y} \frac{\partial^{2} m}{\partial y^{2}}+\left(D_{x y}+D_{y x}\right) \frac{\partial^{2} m}{\partial x \partial y}
\end{aligned}
$$

The second equation of motion, eqn (7), describes the time dependence of the crystal's temperature, $\mathrm{T}$, assumed for simplicity as the same at each point, and $r$ is the temperature scan rate.

In the homogeneous and stationary case, in which the fictitious magnetization, $m$, fulfils the following relations, $\nabla \mathrm{m}=0$ and $\frac{\partial m}{\partial t}=0$, eqn (6) leads to the self-consistent eqn (4) which allowed the computation of the temperaturedependence of the HS fraction of Fig. 9.

In this study, we solved the reaction diffusion eqn (6) and (7) for a 2D square lattice using the finite difference method, ${ }^{41}$ by coarse-graining the time $(d t)$ and the space $(d x, d y)$ using the following step values: $d x=d y=1 \mu m$ and $\mathrm{dt}=0.001 \mathrm{~s}$. The other parameter values used in the model are $\Gamma=0.9 \mathrm{~K}^{-1} \mathrm{~s}^{-1}, \mathrm{D}_{\mathrm{xx}}=20 \mu \mathrm{m}^{2} \mathrm{~s}^{-1}, \mathrm{D}_{\mathrm{yy}}=30 \mu \mathrm{m}^{2} \mathrm{~s}^{-1}, \mathrm{D}_{\mathrm{xy}}$ $=0.005 \mu \mathrm{m}^{2} \mathrm{~s}^{-1}$, and $r=0.2 \mathrm{~K} \mathrm{~s}^{-1}$, for the time scale parameter, the diffusion constants and the scan rate, respectively. For a qualitative comparison to the experimental data, we have chosen rectangular- and triangularshaped lattices having, as far as possible, similar shapes to those of the experiments. Our goal is to check the extent to which this simple model, based on an inhomogeneous dynamical mean-field theory, is capable of capturing the essential aspects of the spatiotemporal features observed in the experimental section.

\section{Spatiotemporal behavior of the HS fraction:}

Eqn (6) and (7) are solved numerically for both cooling and heating processes. The results are summarized in Fig. 10 , which shows a set of selected snapshots, showing the spatiotemporal distribution of the HS fraction, in the course of the phase transition. There are red regions (respectively, blue) that correspond to the HS (respectively, 
LS) phase. The animations displaying the propagation of the HS/LS interface are given in movies SM5-SM9 of the ESI. +

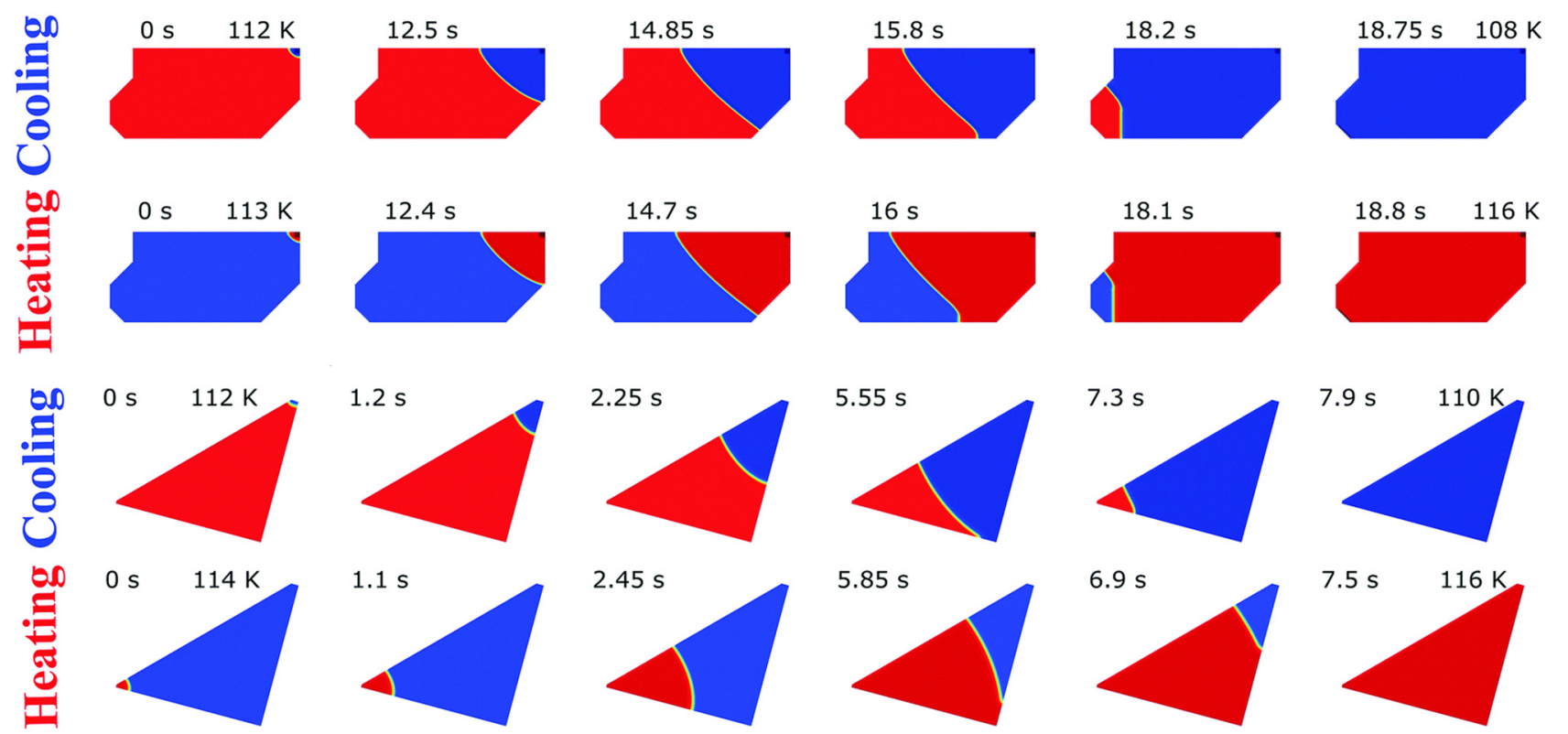

Fig. 10 Selected snapshots showing the spatiotemporal behaviors during the spin transition along the heating and cooling processes. Data obtained with a scan rate $r=0.2 \mathrm{~K} \mathrm{~s}^{-1}$. The red (resp. blue) region corresponds to the HS (resp. LS) phase.

It is easily seen in Fig. 10 and the related movies SM5-SM8 of the ESI, \pm that the HS/LS interface changes its shape during the transition, in excellent agreement with the experimental data of Fig. 5a and experimental movies SM1SM4. In order to fit the experimental border conditions, we imposed the interface to be perpendicular to the edges of the crystal. At this end, at each point of the interface connecting with the lattice border, the relation, $\vec{\nabla}_{n_{\mathrm{HS}}} \cdot \vec{s}=0$ should be fulfilled.

Here, $\vec{\nabla}_{n_{\mathrm{HS}}}$ is the gradient of the HS fraction on a considered point belonging to the HS-LS interface, whose unique non-zero component is perpendicular to the interface line, and $\vec{s}$ is the unit surface vector, normal to the crystal surface or edge. As a result, the interface optimizes its shape during the propagation process so as to minimize its length under the conditions of anisotropic diffusion constant introduced in the equation of motion (8). It is important to mention that using an isotropic diffusion $\left(D_{x x}=D_{y y}=D\right.$ and $\left.D_{x y}=D_{y x}=0\right)$ constant yields an interface behavior that doesn't fit the experimental results. Indeed, in this situation, instead of the inclined HS/LS interface observed in the experiment, we obtain an interface that quickly re-orientates during the process to become straight and perpendicular to the direction of propagation, as shown in movie SM9 of the ESI. t The anisotropic diffusion can be justified by the anisotropic elastic distortion of the unit cell when the system switches from LS to HS, as already discussed in ref. 22 and 42.

Let us now discuss more precisely the details of the simulated interface propagation in the case of the rectangular lattice. The series of snapshots on the top of Fig. 10, depicts the dynamics of the phase transition in the cooling process (HS $\rightarrow L S$ ) starting at temperature $T=112 \mathrm{~K}$ (compare Fig. 9), with a scan rate $r=0.2 \mathrm{~K} \mathrm{~s}^{-1}$. Here, the blue (resp. red) surface represents the stable LS (resp. HS) state. Due to the deterministic character of the spatiotemporal equations of motion $(6,7)$ used in this approach, a small LS nucleus (two or three sites at maximum) is injected in the top right corner of the lattice to initiate the reaction. Experimentally, the nucleation point emerges spontaneously as a result of temperature fluctuations or gradients or due to the presence of defects around the surface. Once the transformation started, its behavior is deterministic. We observe that due to the lattice geometry, initially the interface adopts a circular shape. When it reaches corner 2 (after $12.5 \mathrm{~s}$ ), at which the geometry of the lattice changes, the interface starts a new orientation in order to satisfy the new border conditions imposed by the direction of the lattice edges. Subsequently, at corner 3, a new change occurs, and because the upper part of the interface is ahead of the lower one, the front adopts a sigmoidal shape in very good agreement with the experimental data of Fig. $5 \mathrm{a}$. The heating process for the rectangular case was performed at temperature $\mathrm{T}=113$ $\mathrm{K}$ and led to similar observations to those of the cooling mode. 
In the second part of Fig. 10, we present the simulated spatiotemporal transformation of the triangularly-shaped crystal, for which we studied the dynamics of the phase transition on heating and cooling at the respective temperatures $\mathrm{T}=114 \mathrm{~K}$ and $112 \mathrm{~K}$. Similarly, the interface's shape follows the whole geometry of the lattice in good agreement with the experimental results of Fig. $5 \mathrm{a}$.

In Fig. S5 (ESI ${ }^{\dagger}$ ), we report the successive x-coordinate of the center of the HS-LS front through the crystal along

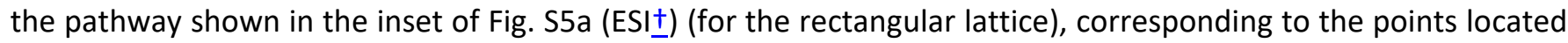
in the middle HS-LS interface. We can easily notice the parabolic behavior of the time-dependence of the interface position, with the presence of an incubation regime, in the first period of the propagation mechanism, the interface spreads slowly, and then it gradually accelerates until the end of the process where the velocity reaches its maximum value. A similar behavior of the HS-LS interface is observed for the cooling process. These results are in very good qualitative agreement with the experimental findings displayed in Fig. 5 . In contrast, Fig. S5b (ESI†), presenting the time evolution of the $x$-coordinate of the center of the HS-LS interface during the spin transition in the triangle, reveals a clear monotonous behavior leading in a large part of the process (except in the end) to a uniform motion.

Fig. 11, summarizes the spatiotemporal behavior of the field velocity, drawn on the contour plot of each interface for both crystals for the HS to LS transition. The global change of the orientation of the field velocity according to the interplay between the interface and the geometry of the lattice is in very good agreement with the experimental results of Fig. 5a. Interestingly, for both shapes (rectangle and triangle), an enhancement of the magnitude of velocity is observed when the system reaches the final stage of the transformation, as a result of the interaction between the interface and the opposite side of the crystal's edge.
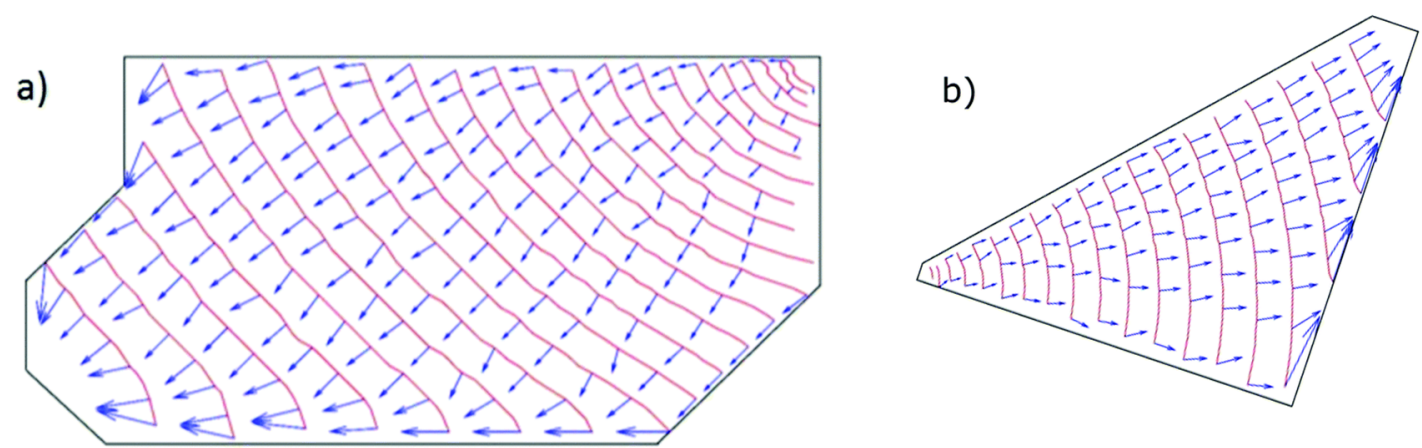

Fig. 11 The spatial distribution of the instantaneous velocity field during the heating (LS $\rightarrow$ HS) process for the (a) $R$ and $(b)$ $T$ lattices. Notice the good agreement with the experimental data of Fig. 6.

To make a complete comparison with the experimental data, we have also studied the local kinetics of the transformation through the analysis of the temporal evolution of the magnetization at a given position of the lattice during the front propagation (on cooling) in the rectangular lattice. The results are illustrated in Fig. S6 of the ESI. \pm At the beginning, the lattice was prepared in the HS state, i.e. $m=+1$ at all sites, except in the corner. This results in a sudden drop of the HS fraction, in the first regime of Fig. S6a (ESII), in which the lattice relaxes to the stationary mean-field solution $\mathrm{m}=0.7554$ of $\underline{\mathrm{Fig} .9}$, at temperature $\mathrm{T}=112 \mathrm{~K}$. The time-dependence of the HS fraction in this first fast homogeneous regime is presented in detail in Fig. S6b (ESI $t$ ), and clearly follows an exponential trend, contrasting with the second regime of interface propagation, depicted in Fig. S6c (ESI + ), which is sigmoidal as a result of the system's non-linearity.

\section{Conclusions}

This work provided the spatiotemporal aspects of the thermo-induced spin transition of [Fe(2pytrz $\left.)_{2}\left\{\mathrm{Pd}(\mathrm{CN})_{4}\right\}\right] \cdot 3 \mathrm{H}_{2} \mathrm{O}$ single crystals. We have chosen two single crystals with different shapes that undergo a firstorder spin transition between the HS-HS and incomplete HS-LS plateau with nucleation and single domain growth processes. Experimental investigations were performed by means of optical microscopy based on the color change of the single crystals between the high-and low-temperature phases. The analysis of the temperature-dependence of the spatially-averaged optical density change upon the spin transition revealed hysteresis loops with quite similar 
switching temperatures of $\sim 152 \mathrm{~K}$ and $\sim 162 \mathrm{~K}$ on cooling and heating, respectively. Furthermore, these temperatures were revealed to be in good agreement with those obtained by magnetic $(160.0,163.5 \mathrm{~K})$ and DSC $(158.7,160.6 \mathrm{~K})$ measurements. In addition, we estimated the velocity of the front's propagation in the range of $\sim 2-8 \mu \mathrm{m} \mathrm{s}^{-1}$. However, the new evidence provided by the present study shows the existence of an interplay between the interface's shape and that of the crystal, thanks to the constraints of contact angle to which the front of transformation is subjected. Subsequently, we determined the field velocity which confirmed the self-adaptation process of the interface's velocity so as to minimize its elastic energy as well as to adapt the change of the border conditions.

Due to the reduced volume change at the transition of these crystals, their description using a theoretical approach based on a reaction diffusion model, proved to be very accurate. Indeed, this simple approach allowed an analytical spatiotemporal equation of motion to be built for the local high-spin fraction, which was solved for different configuration shapes of the crystal, similar to those of the experiment. The most important features of the observed spatiotemporal kinetics were reproduced, including the self-adaptation of interface shape, orientation and velocity during the spin transition. On top of that, this work opens the way to several extensions, for example, imaging by optical microscopy of the light-induced transformation of crystals, including the effect of crystal shape on the cooperative relaxation of the metastable photo-induced HS state, whose spatiotemporal properties should also be demystified.

\section{Conflicts of interest}

There are no conflicts to declare.

\section{Acknowledgements}

This work was financially supported by CNRS ("Centre National de la Recherche Scientifique"), the universities of Versailles and Brest, the French "Ministère de la Recherche" and the "Agence Nationale de la Recherche" (ANR Project BISTA-MAT: No.ANR-12-BS07-0030-01) and the France-Japan LIA (International Associate Laboratory). We also acknowledge Mouhamadou Sy and Guillaume Bouchez for the technical assistance with the optical microscopy measurements, as well as Bilel Filali for helping with the simulation work.

\section{Notes and references}

1. P. Gütlich and H. A. Goodwin, Topics in Current Chemistry, Spin Crossover in Transition Metal Compounds I-III, Springer, Heidelberg, Berlin, 2004, pp. 233-235

2. P. Gütlich, A. Hauser and H. Spiering, Angew. Chem., Int. Ed. Engl., 1994, 33, 2024-2054

3. C. P. Köhler, R. Jakobi, E. Meissner, L. Wiehl, H. Spiering and P. Gütlich, J. Phys. Chem. Solids, 1990, 51, 239247

4. E. König, Complex Chemistry, Springer Berlin Heidelberg, Berlin, Heidelberg, 1991, pp. 51-152

5. A. Bousseksou, N. Negre, M. Goiran, L. Salmon, J.-P. Tuchagues, M.-L. Boillot, K. Boukheddaden and F. Varret, Eur. Phys. J. B, 2000, 13, 451-456

6. A. Hauser, J. Jeftić, H. Romstedt, R. Hinek and H. Spiering, Coord. Chem. Rev., 1999, 190, 471-491

7. T. Kambara, J. Phys. Soc. Jpn., 1980, 49, 1806-1811

8. N. Sasaki, J. Chem. Phys., 1981, 74, 3472

9. S. W. Biernacki and B. Clerjaud, Phys. Rev. B: Condens. Matter Mater. Phys., 2005, 72

10. G. D'Avino, A. Painelli and K. Boukheddaden, Phys. Rev. B: Condens. Matter Mater. Phys., 2011, 84, 104119

11. M. Castro, O. Roubeau, L. Piñeiro-López, J. A. Real and J. A. Rodríguez-Velamazán, J. Phys. Chem. C, 2015, $119,17334-17343$

12. Y. De Gaetano, E. Jeanneau, A. Y. Verat, L. Rechignat, A. Bousseksou and G. S. Matouzenko, Eur. J. Inorg. Chem., 2013, 1015-1023

13. S. Pillet, J. Hubsch and C. Lecomte, Eur. Phys. J. B, 2004, 38, 541-552

14. S. Gawali-Salunke, F. Varret, I. Maurin, C. Enachescu, M. Malarova, K. Boukheddaden, E. Codjovi, H. Tokoro, S. Ohkoshi and K. Hashimoto, J. Phys. Chem. B, 2005, 109, 8251-8256

15. V. Mishra, R. Mukherjee, J. Linares, C. Balde, C. Desplanches, J. F. Letard, E. Collet, L. Toupet, M. Castro and F. Varret, Inorg. Chem., 2008, 47, 7577-7587 
16. F. Varret, C. Chong, A. Goujon and K. Boukheddaden, J. Phys.: Conf. Ser., 2009, 148, 012036

17. A. Goujon, F. Varret, K. Boukheddaden, C. Chong, J. Jeftić, Y. Garcia, A. D. Naik, J. C. Ameline and E. Collet, Inorg. Chim. Acta, 2008, 361, 4055-4064

18. C. Chong, H. Mishra, K. Boukheddaden, S. Denise, G. Bouchez, E. Collet, J.-C. Ameline, A. D. Naik, Y. Garcia and F. Varret, J. Phys. Chem. B, 2010, 114, 1975-1984

19. A. Slimani, F. Varret, K. Boukheddaden, C. Chong, H. Mishra, J. Haasnoot and S. Pillet, Phys. Rev. B: Condens. Matter Mater. Phys., 2011, 84, 094442

20. F. Varret, A. Slimani, K. Boukheddaden, C. Chong, H. Mishra, E. Collet, J. Haasnoot and S. Pillet, New J. Chem., 2011, 35, 2333

21. C. Chong, A. Slimani, F. Varret, K. Boukheddaden, E. Collet, J. C. Ameline, R. Bronisz and A. Hauser, Chem. Phys. Lett., 2011, 504, 29-33

22. M. Sy, F. Varret, K. Boukheddaden, G. Bouchez, J. Marrot, S. Kawata and S. Kaizaki, Angew. Chem., 2014, 126, 7669-7672

23. M. Nishino, C. Enachescu, S. Miyashita, P. A. Rikvold, K. Boukheddaden and F. Varret, Sci. Rep., 2011, 1, 162

24. A. Slimani, K. Boukheddaden, F. Varret, M. Nishino and S. Miyashita, J. Chem. Phys., 2013, 139, 194706

25. A. Slimani, K. Boukheddaden and K. Yamashita, Phys. Rev. B: Condens. Matter Mater. Phys., 2015, 92, 014111

26. R. Traiche, M. Sy, H. Oubouchou, G. Bouchez, F. Varret and K. Boukheddaden, J. Phys. Chem. C, 2017, 121, 11700

27. A. Slimani, F. Varret, K. Boukheddaden, D. Garrot, H. Oubouchou and S. Kaizaki, Phys. Rev. Lett., 2013, 110, 087208

28. R. H. Wiley and A. J. Hart, J. Org. Chem., 1953, 18, 1368-1371

29. E. Milin, V. Patinec, S. Triki, E.-E. Bendeif, S. Pillet, M. Marchivie, G. Chastanet and K. Boukheddaden, Inorg. Chem., 2016, 55, 11652-11661

30. F. Varret, C. Chong, A. Slimani, D. Garrot, Y. Garcia and A. D. Naik, Spin-Crossover Materials, John Wiley \& Sons Ltd, 2013, ch. 16, pp. 425-441

31. M. Nishino, C. Enachescu, S. Miyashita, K. Boukheddaden and F. Varret, Phys. Rev. B: Condens. Matter Mater. Phys., 2010, 82, 020409

32. C. Enachescu, L. Stoleriu, A. Stancu and A. Hauser, Phys. Rev. Lett., 2009, 102, 257204

33. A. Slimani, K. Boukheddaden, F. Varret, H. Oubouchou, M. Nishino and S. Miyashita, Phys. Rev. B: Condens. Matter Mater. Phys., 2013, 87, 014111

34. M. Nishino, T. Nakada, C. Enachescu, K. Boukheddaden and S. Miyashita, Phys. Rev. B: Condens. Matter Mater. Phys., 2013, 88

35. R. Traiche, H. Oubouchou, M. Zergoug and K. Boukheddaden, Phys. B, 2017, 516, 77-84

36. K. Boukheddaden, A. Slimani, M. Sy, F. Varret, H. Oubouchou and R. Traiche, in Magnetic Structures of 2D and 3D Nanoparticles - Properties and Applications, ed. F. Jean-Claude Levy, Physical Properties of 2D SpinCrossover Solids from an Electro-Elastic Description: Effect of Shape, Size, and Spin-Distortion Interactions, Université Paris-Diderot, Pan Stanford Publishing, Pan Stanford Publishing, CRC Press, 2016 edn, 2016, ch. 9, p. 70

37. C. Enachescu, M. Nishino, S. Miyashita, K. Boukheddaden, F. Varret and P. A. Rikvold, Phys. Rev. B: Condens. Matter Mater. Phys., 2015, 91, 104102

38. M. Paez-Espejo, M. Sy, F. Varret and K. Boukheddaden, Phys. Rev. B: Condens. Matter Mater. Phys., 2014, 89,024306

39. K. Boukheddaden, I. Shteto, B. Hôo and F. Varret, Phys. Rev. B: Condens. Matter Mater. Phys., 2000, 62, 14796-14805

40. A. Bousseksou, J. Nasser, J. Linares, K. Boukheddaden and F. Varret, J. Phys., 1992, 2, 1381-1403

41. M. Sy, D. Garrot, A. Slimani, M. Páez-Espejo, F. Varret and K. Boukheddaden, Angew. Chem., Int. Ed., 2016, $55,1755-1759$

42. M. Paez-Espejo, M. Sy and K. Boukheddaden, J. Am. Chem. Soc., 2016, 138, 3202-3210 